\title{
PLACE OF DEATH AND ACCESS TO HOME CARE SERVICES: ARE CERTAIN PATIENT GROUPS AT A DISADVANTAGE?
}

\author{
G. E. GRANDE, ${ }^{*}$ J. M. ADDINGTON-HALL ${ }^{2}$ and C. J. TODD ${ }^{1}$ \\ ${ }^{1}$ Department of Community Medicine, GPPCRU, Institute of Public Health, University of Cambridge, \\ University Forvie Site, Robinson Way, Cambridge CB2 2SR, U.K. and ${ }^{2}$ Department of Palliative Care \\ and Policy, King's College School of Medicine and Dentistry/St. Christopher's Hospice, London \\ SE5 9PJ, U.K.
}

\begin{abstract}
Research indicates that fewer people are able to die at home than would wish to do so. Furthermore the ability to die at home is unequally distributed depending on patient characteristics. Unless factors associated with home deaths are identified and interventions are targeted accordingly, further general improvements in care support may only help those already at an advantage. This paper reviews research investigating the relation between patient characteristics and home deaths and considers whether these variables influence place of death because they are associated with differential access to services, focusing on access to palliative home care. Patients with informal carer support were both more likely to die at home and to access palliative home care. Provision of home care did not remove the dependence on informal carers in achieving home death, however. An important target in improving home death rates is therefore better support for informal carers overall. Older patients were both less likely to die at home and to access home care. Once in home care they no longer were less likely to die at home. Although age related needs require consideration, improved access to home care is therefore likely to increase home deaths for older people. Women were less likely to die at home than men, yet younger women may be more likely to access home care. There is some evidence to suggest that men were less efficient as carers, which may help explain why women were less likely to achieve home deaths, while making their referral to home care more likely. While home care may help redress the gender imbalance, men may also need to be encouraged and enabled to take on the carer role. Cancer patients in higher socioeconomic groups were both more likely to die at home and to access home care. Hence home deaths may increase by improving access for lower socioeconomic groups to the services available. (C) 1998 Elsevier Science Ltd. All rights reserved
\end{abstract}

Key words - place of death, palliative care, home care, terminal care

\section{INTRODUCTION}

Approximately $25 \%$ of people in the U.K. currently die at home (Seale and Cartwright, 1994) and lower percentages have been cited for cancer deaths in U.S.A. and Australia (Hunt and McCaul, 1996). However, research suggests that death at home is preferred by one half or more of terminally ill patients (Dunlop et al., 1989; Townsend et al., 1990), by a majority of the general public (Charlton, 1991; Toscani et al., 1991; Ashby and Wakefield, 1993) and primary care professionals (Cartwright, 1991). Bereaved informal carers are also more likely to state that the place of death was right (Ward, 1987; Addington-Hall et al., 1991) and suffer less distress (Catalan-Fernandez et al., 1991) if the patient died at home rather than in hospital. There is therefore an imbalance between what primary care support is able to provide and what patients, carers, health professionals and the public want. However, access to palliative home care ser-

*Author for correspondence. vices and ability to die at home is unequally distributed and further general increases in palliative care support may mainly help those who are already at an advantage. This paper summarises what is known about differences in death at home and access to palliative home care in order to identify target groups for further research and intervention.

The paper reviews research relating to home deaths and access to palliative home care for adults. Studies were identified through the databases Medline Express 1968-1997 and SERLINE on SilverPlatter 1997 and by manually following up references cited in identified papers. The search terms used were "place" or "location of death" and "terminal" or "palliative" "home care" or "domiciliary care". The review was limited to English language papers and therefore mainly contains research conducted in the U.K., the U.S.A. and Australia. However, papers from Canada, Italy, Sweden, Israel and Switzerland are included. The results are therefore restricted to the historico-cultural context of Europe, North America and Australia in recent decades. Aries (1981) paints 


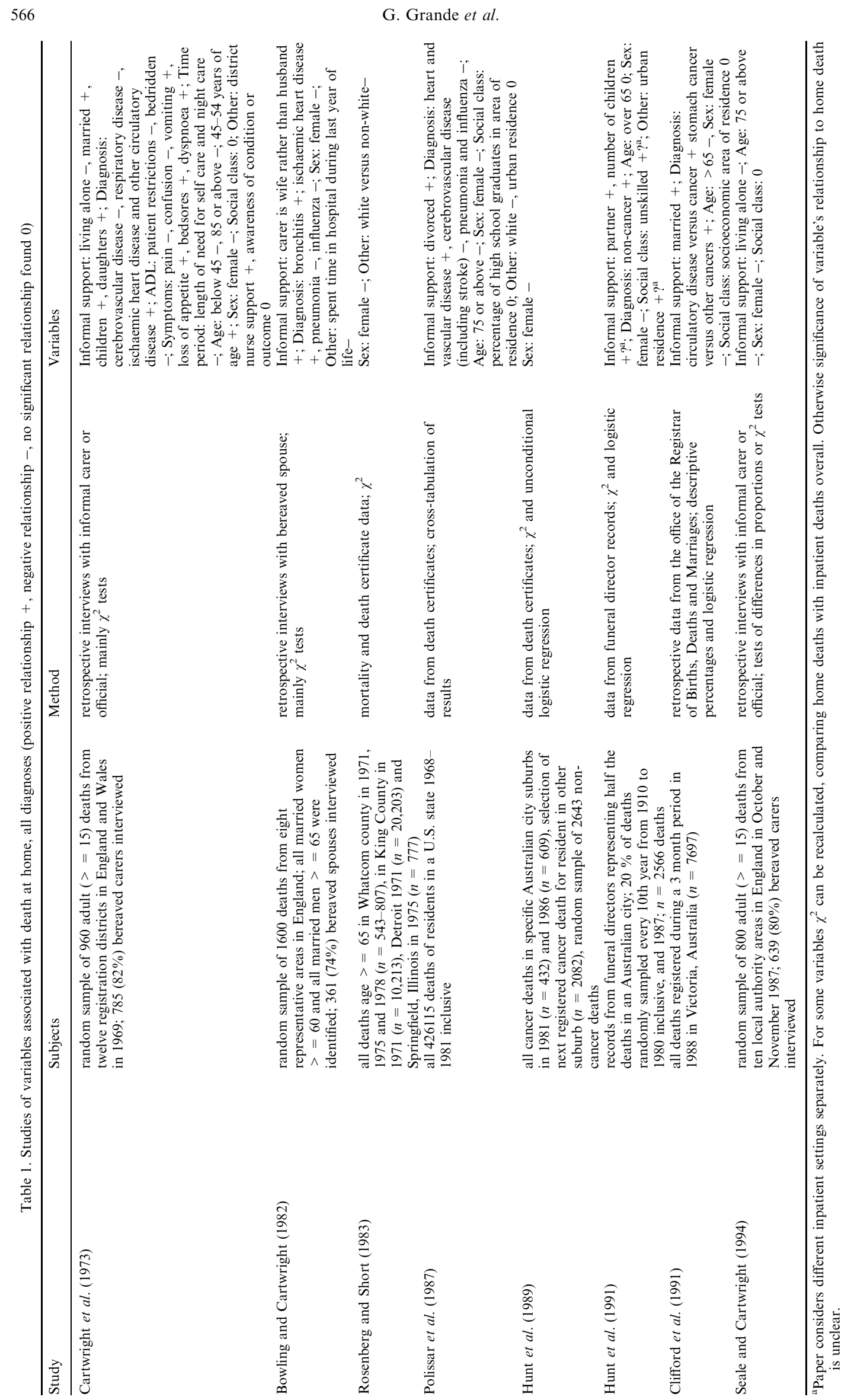


Place of death and access to home care services

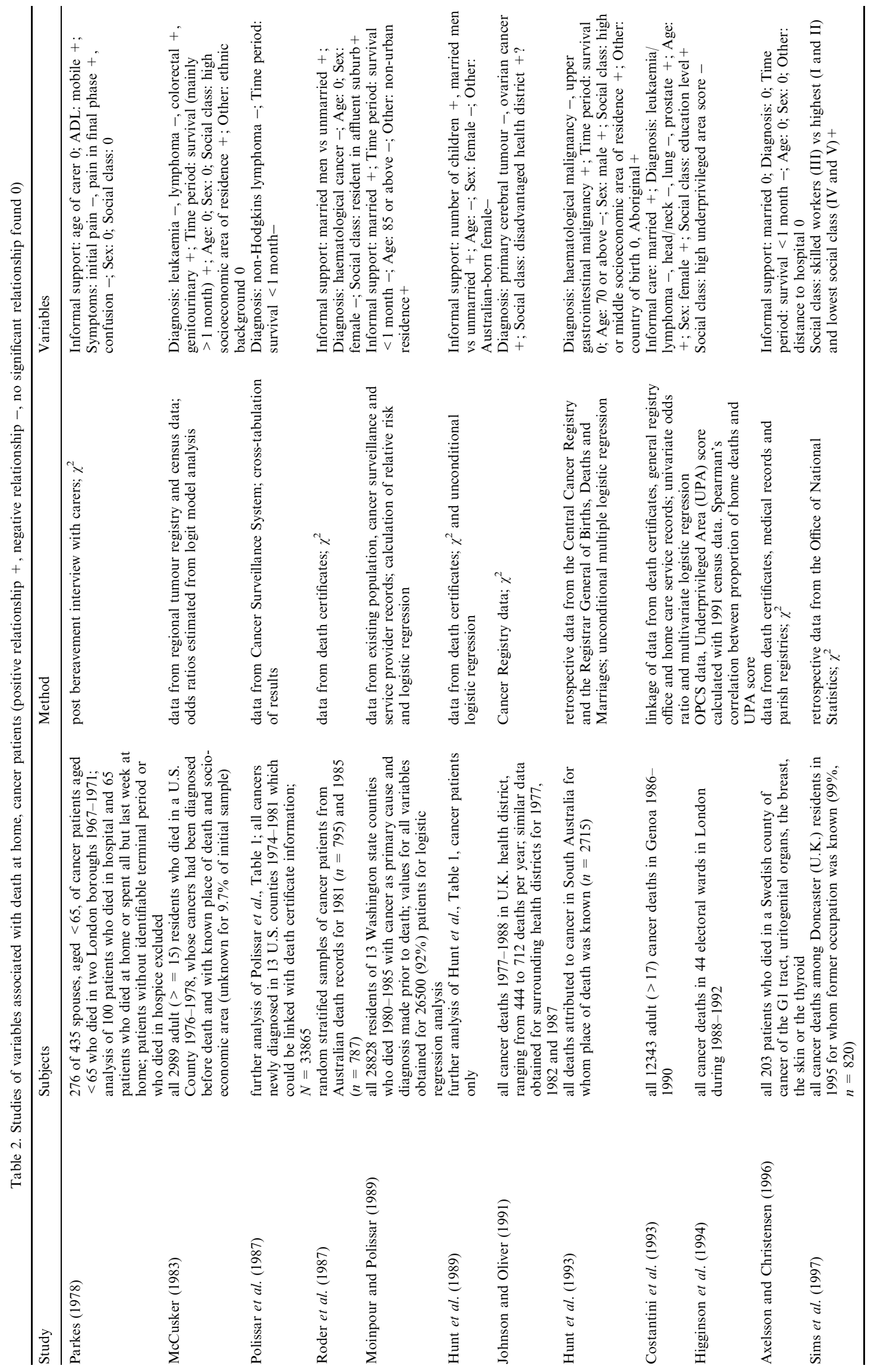




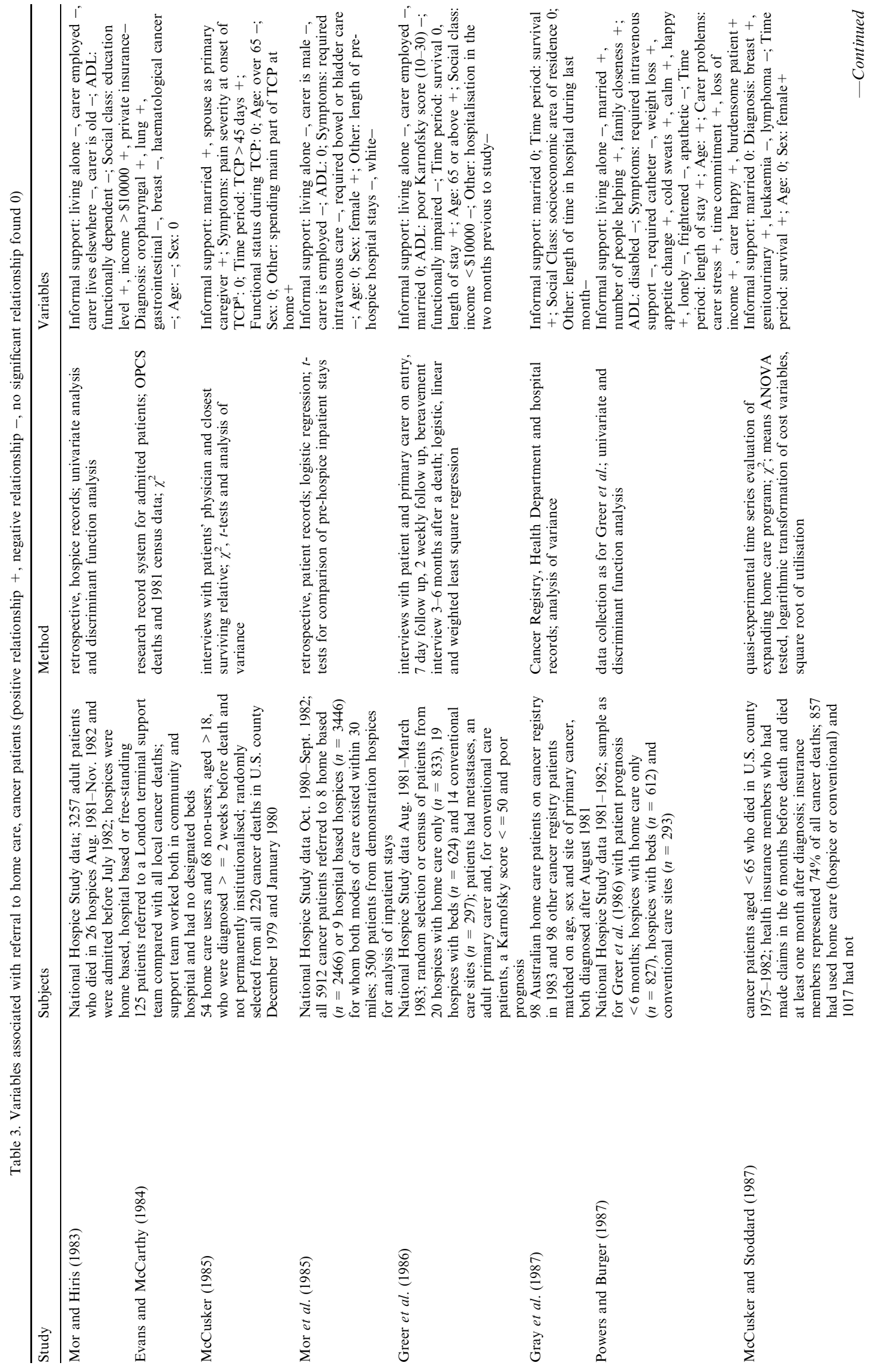



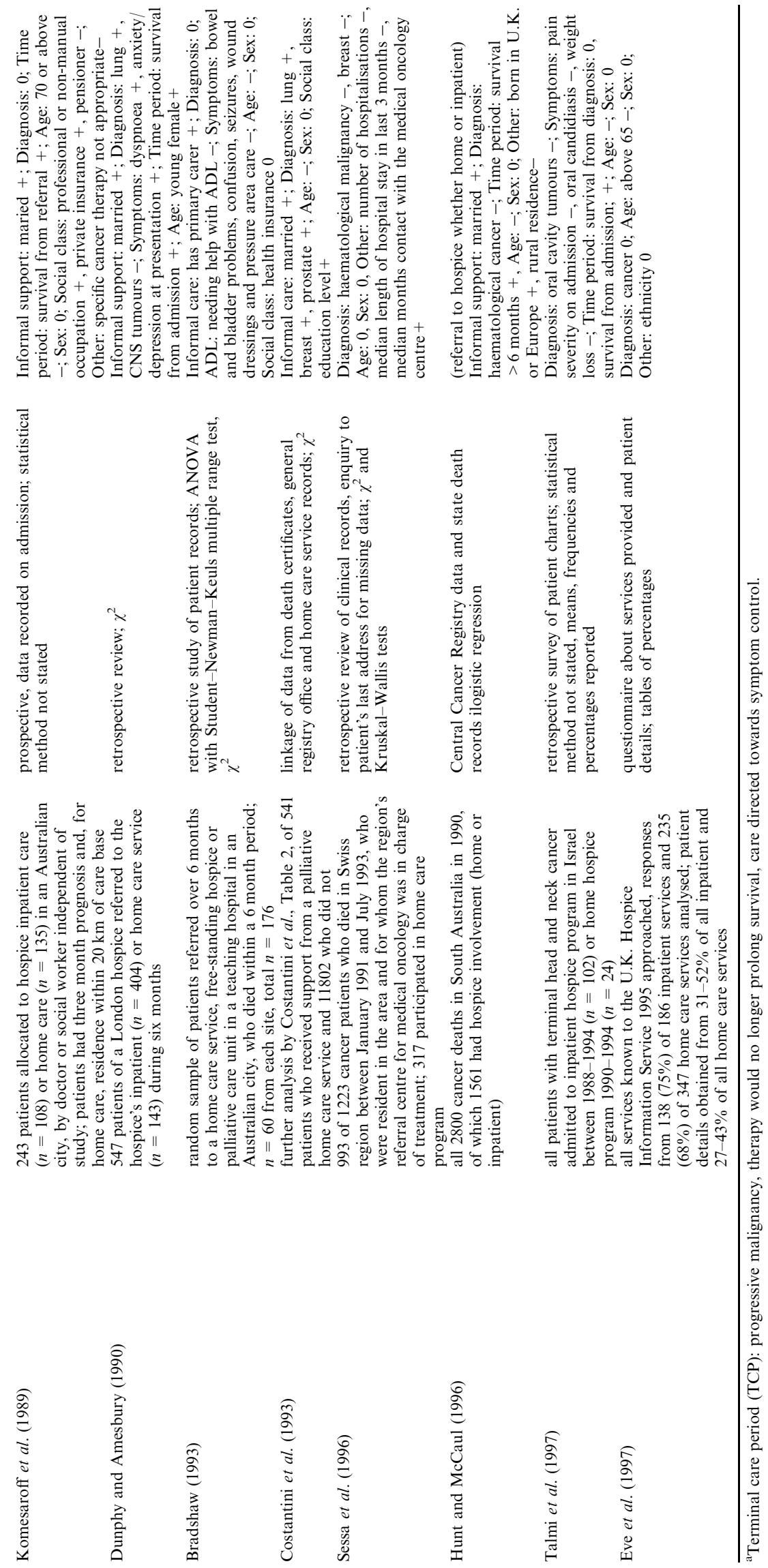


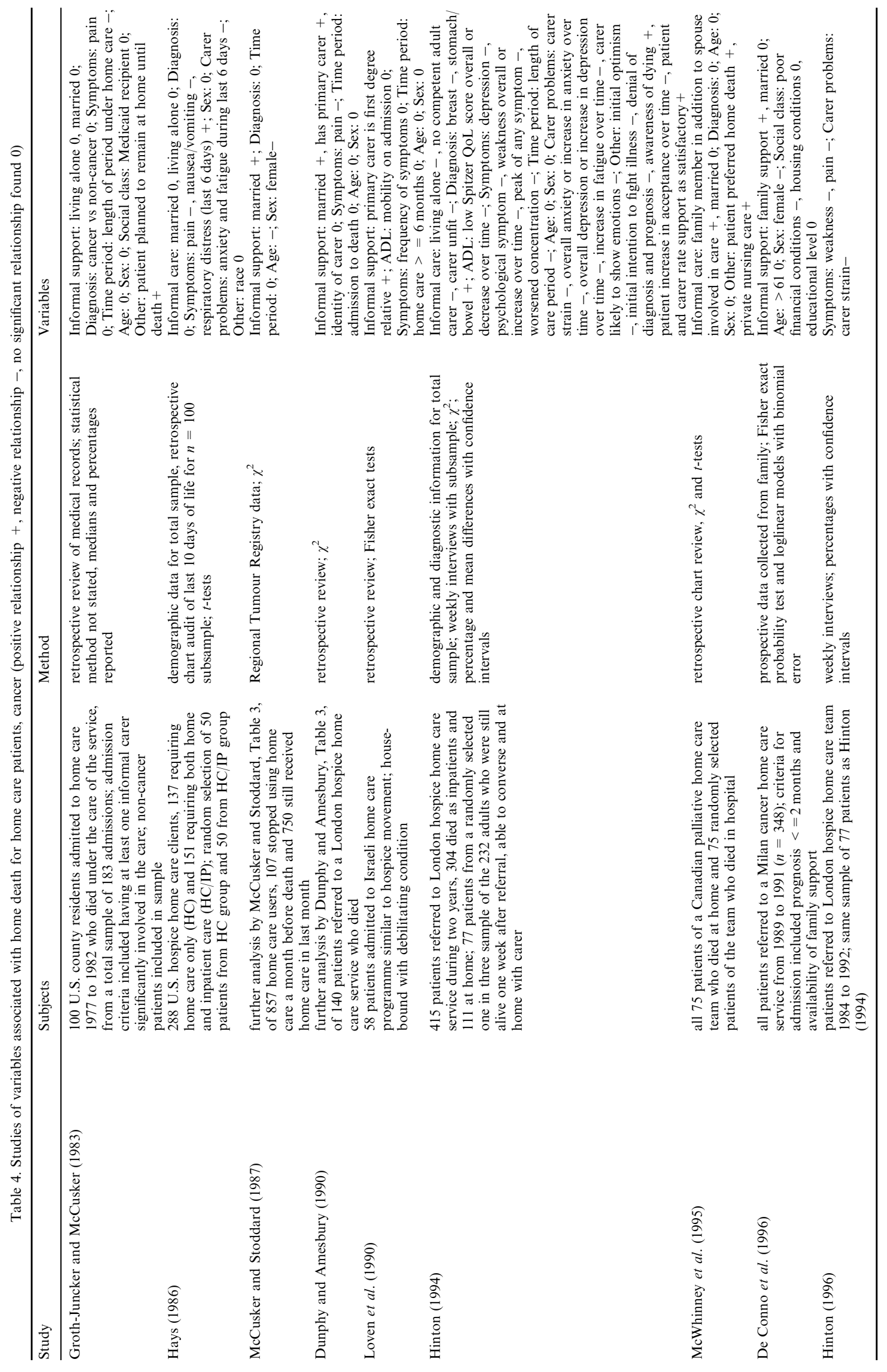


quite different pictures of death during the development of western civilisation, and death in other cultures today may also be quite different in form and meaning to the "hidden death" of the late twentieth century "European" model. The review excluded studies conducted on patients identified through hospital specialist oncology services as these patients probably represent a selective subset of the cancer population, the particular characteristics of which were difficult to determine. For instance, only $38 \%$ of lung cancer patients in a U.K. region had been in contact with oncology services (Sanderson et al., 1992). All other studies which consider patient or carer variables associated with death at home or access to palliative home care were included, regardless of study methodology.

The paper first identifies characteristics of patients dying at home for the population in general and cancer patients in particular. It then considers characteristics of cancer patients referred to palliative home care teams and place of death for cancer patients under home care, with the aim of assessing whether cancer patients with certain characteristics tend to die at home because they have better access to palliative home care, rather than because of their characteristics per se. Similar literature on home care for non-cancer patients was not found using our search strategy. The home setting is compared to other care settings overall, but data relating to differences in proportions of deaths in different inpatient settings are not considered. Tables 1-4 include all variables investigated in the studies relating to palliative home care access and home deaths.

\section{STUDIES INVESTIGATING PLACE OF DEATH BOTH FOR CANCER AND NON-CANCER PATIENTS}

Studies identified (Table 1) suggest the importance of informal carer support in facilitating home deaths. Patients who lived with someone, were married or had a partner were more likely to die at home than those who did not (Cartwright et al., 1973; Clifford et al., 1991; Hunt et al., 1991; Seale and Cartwright, 1994). Only one study found that divorced patients were more likely to die at home than others (Polissar et al., 1987). The identity of the informal carer mattered. If the wife was the carer, death at home was more likely than if the husband was the carer (Bowling and Cartwright, 1982). The availability of children for support appeared more important than the presence of a spouse and the presence of daughters more important than that of sons (Cartwright et al., 1973).

Men were more likely to die at home than women (Cartwright et al., 1973; Bowling and Cartwright, 1982; Rosenberg and Short, 1983; Polissar et al., 1987; Hunt et al., 1989, 1991; Clifford et al., 1991; Seale and Cartwright, 1994). As women tend to live longer than men, they are more likely to be frail and widowed by the time they die. This is reflected in women's greater likelihood of dying in nursing or residential homes than men (Cartwright et al., 1973; Polissar et al., 1987; Hunt et al., 1989, 1991; Clifford et al., 1991; Seale and Cartwright, 1994). However, as seen above, home death was more likely when wives and daughters were the carers rather than husbands and sons, which suggest that gender roles also play a part.

According to data from the 1960 s, patients with fewer restrictions and a shorter care period were more likely to die at home than their counterparts, while length of incontinence rather than incontinence per se was associated with hospital deaths (Cartwright et al., 1973). Patients who died in hospital were more likely to have suffered pain and confusion, while home death was associated with vomiting, loss of appetite, bedsores and dyspnoea (Cartwright et al., 1973).

Home deaths were least likely for patients who died from cerebrovascular disease (Cartwright et al., 1973; Polissar et al., 1987), pneumonia and influenza (Bowling and Cartwright, 1982; Polissar et al., 1987), all three of which are often associated with old age. Home deaths were most likely for those with heart and other vascular disease (Cartwright et al., 1973; Bowling and Cartwright, 1982; Polissar et al., 1987; Clifford et al., 1991), which often imply a sudden death. Cartwright et al.'s (1973) data suggest that patients with respiratory disease were among those least likely to die at home, while Bowling and Cartwright's (1982) report that patients with bronchitis were among those most likely. Although these two disease categories overlap they are not the same. Hunt et al.'s (1991) findings indicate that home death was less likely for cancer patients than for non-cancer patients in Australia during this century.

Polissar et al. (1987), Clifford et al. (1991) and Seale and Cartwright (1994) found that patients aged 75 years and above were less likely to die at home than other adults. Cartwright et al.'s (1973) earlier study found home deaths to be least likely for patients under 45 years of age and patients aged 85 and above, while Hunt et al. (1991) found no relationship between age and home deaths in Australia in the time period 1910 to 1987 . Social class did not appear to show a relationship with home deaths (Cartwright et al., 1973; Polissar et al., 1987; Hunt et al., 1991; Clifford et al., 1991; Seale and Cartwright, 1994). Patients who died in hospital were more likely to have spent time in hospital in the last year of life than patients dying at home (Bowling and Cartwright, 1982), while those who died at home had greater district nurse input (Cartwright et al., 1973). However, level of primary or secondary care input may have been an effect of place of death itself. 


\section{STUdies INVESTIGATING CANCER PATIENTS' PLACE OF} DEATH

Seale (1991) notes that cancer patients are different from other disease groups. They tend to die younger and are therefore more likely to have a living spouse and other living relatives. Age related symptoms such as mental confusion and long term disability are less common. Furthermore, the incidence, duration, intensity and type of symptoms follow a different course and a terminal phase can be more easily distinguished. The variables related to place of death for cancer patients may therefore be different to those for the rest of the population.

Studies reviewed (Table 2) again indicate that being married is positively associated with death at home, either for both sexes (Moinpour and Polissar, 1989; Costantini et al., 1993) or, as found in Australia, for males only (Roder et al., 1987; Hunt et al., 1989). Home death was positively related to number of children for both sexes (Hunt et al., 1989). Axelsson and Christensen (1996) found no effect of marriage on home deaths. However, the number of home deaths in their study was small $(n=24)$. Australian women were less likely to die at home than Australian men (Roder et al., 1987; Hunt et al., 1989). However, Costantini et al. (1993) found the reverse for Italian women.

Moinpour and Polissar (1989), Hunt et al. (1989) and Hunt et al. (1993) report that older patients were less likely to die at home than younger patients. However, Costantini et al. (1993) found that it was the older patients who were most likely to die at home. Parkes (1978), McCusker (1983) and Axelsson and Christensen (1996) found no effect of age on home deaths.

Patients who were diagnosed less than a month before death were less likely to die at home (McCusker, 1983; Polissar et al., 1987; Moinpour and Polissar, 1989; Axelsson and Christensen, 1996). This may reflect a high incidence of hospital tests and attempts at treatment in the month following cancer diagnosis. Increase in interval between diagnosis and death beyond a month appeared to have little effect on home deaths.

Parkes (1978) data suggest that patients who died in hospital were less mobile, more likely to suffer confusion and more likely to suffer pain initially than patients remaining at home. However, in the final phase of illness, patients who remained at home were likely to experience more pain.

Diagnoses especially associated with home deaths were gastrointestinal and genitourinary cancer (McCusker, 1983; Clifford et al., 1991; Johnson and Oliver, 1991; Costantini et al., 1993; Hunt et al., 1993). Patients with haematological cancers were more likely to die in acute care (McCusker, 1983; Polissar et al., 1987; Roder et al., 1987; Costantini et al., 1993; Hunt et al., 1993) presumably due to a greater amount of hospital treatment. Patients with head/neck or lung cancer (Costantini et al., 1993) and primary cerebral tumours (Johnson and Oliver, 1991) also tended to die in hospital. The latter would be associated with confusion, a variable which is associated with hospital death (Cartwright et al., 1973), but it is less clear why the other sites may result in hospitalisation.

Several studies show that cancer patients who had higher education (Costantini et al., 1993) or were living in a higher socioeconomic area of residence (McCusker, 1983; Roder et al., 1987; Hunt et al., 1993; Higginson et al., 1994) had a greater likelihood of dying at home than their counterparts. McCusker's (1983) U.S. study showed this trend to be reversed only for people in areas sufficiently deprived to warrant reimbursement of home care services. While Higginson et al. (1994) investigated both patients with cancer and those with circulatory disorders, socioeconomic differences were found for cancer patients only. Data from one recent study (Sims et al., 1997) suggests that those in skilled occupations were more likely to die at home compared both to higher and lower occupational groups. However, the lower occupational groups, representing $61 \%$ of the sample, were considerably more likely to die in hospital and less likely to die in a hospice compared with the other groups. Thus the lower occupational groups appear at a disadvantage both in terms of home death and access to cancer related services. Johnson and Oliver (1991) reported that the district in their investigation was more disadvantaged than surrounding districts, yet had a higher proportion of home deaths. However, they did not conduct a formal investigation of this relationship. Parkes (1978) found no effect of social class.

In summary the importance of having support from a partner, children or other primary carer is illustrated both for studies of the general population and those relating to cancer patients. The strain placed on primary carers in the form of patients' care requirements also has an impact on home deaths. This illustrates the dependency on the primary carer in achieving home deaths.

Overall males appeared more likely to die at home and older people less likely to do so. Patients who are female and old are therefore at a disadvantage if death at home is the desired aim. This may partly be related to availability of primary care support as females tend to die at a higher age than men, which means that presence of a primary carer is less likely (Seale and Cartwright, 1994). The one study which found females and old people to have greater likelihood of home death was Italian (Costantini et al., 1993), which suggests that it is not simply gender or age per se which is important, but the cultural and family context in which patients live. Gender roles are also likely to play a part, as studies of the general population show that home death is more likely if the carer is female. 
Females are probably more likely to be cast in the role as the provider rather than the recipient of care. By encouraging and helping males to take on the role as care providers one may tap a hitherto underused resource.

There were socioeconomic differences in place of death for cancer patients. In contrast, such differences were not found in the studies of deaths from all causes, reviewed in Section 2, or when considering patients with circulatory disease (Higginson et al., 1994). Currently a greater range of support is possible for cancer than for other diseases, e.g. in the form of Marie Curie nursing care in the U.K. or hospice care. The greater range of support available may lead to greater differences between those who are effective in gaining access to support and those who are not, compared to other common, long term diseases. Only Parkes (1978) found no effect of socioeconomic status. However, this was an early study before cancer care services in the U.K. were that well developed. Access may be related to ability to pay for care, at least in the U.S.A., as McCusker's (1983) study showed the effect to be removed if home care service use was reimbursed. Individuals from higher socioeconomic groups may also be more articulate and resourceful in accessing the range of support available locally. Furthermore they may be better able to ensure that they live in a setting with a good level of services available, because they can afford to live in affluent areas, and they may also be more effective in ensuring that support services are established locally. As the socioeconomic differences found mainly relate to socioeconomic area of residence, one needs to establish whether it is the socioeconomic level of the individual or that of their area of residence which is most closely related to place of death, as this would require totally different approaches to amending inequality.

\section{THE EFFECT OF SERVICES ON PLACE OF DEATH}

Referral to palliative home care services is likely to be a contributory factor towards home death. Indeed, users of home care services are more likely to die at home than other patients (Zimmer et al., 1984; Greer et al., 1986; Gray et al., 1987; Komesaroff et al., 1989; Moinpour and Polissar, 1989; Dunphy and Amesbury, 1990; Costantini et al., 1993; Sessa et al., 1996). However, the type of home care matters. Patients receiving home care attached to an inpatient service are considerably less likely to die at home than patients receiving home care not thus attached (Ward, 1987; Smith et al., 1992). Integration with inpatient care may therefore facilitate inpatient admission. It is not clear, however, whether home care with bed attachment may in fact at times be associated with fewer deaths at home than no home care.
Studies suggest that it is the particular support service per se which facilitates home death rather than the characteristics of the patients referred. A discriminant function analysis of place of death for U.S. hospice patients showed that the variable best able to predict place of death was the type of hospice, i.e. whether it was home care based only or had beds (Mor and Hiris, 1983). Once type of hospice was considered, knowledge of patients' demographic characteristics and support-network added only $1 \%$ to the ability to predict site of death. The case-mix between hospices was found to be less able to predict place of death than the type of hospice. A logistic regression analysis of cancer patients in South Australia (Hunt and McCaul, 1996) showed that place of death was largely determined by whether or not the patient was admitted to a hospice (providing either home or inpatient support). While there were demographic and clinical differences between admitted and non-admitted patients, multivariate analysis showed that these differences had very little effect on the odds of dying in different settings. Finally, a randomised controlled trial of a home care team found that patients assigned to the team had a greater likelihood of dying at home (Zimmer et al., 1984).

Patients with certain characteristics may therefore be more likely to die at home by virtue of being more likely to access services which improve their chances of dying at home. Inequalities in referral to services may therefore account for inequalities in place of death. Two approaches are used to assess this further. First, by considering which patients are referred to palliative home care and checking if the patterns are identical to those relating to place of death. Second, by reviewing place of death of home care patients to ascertain whether the previously observed relationships between patient characteristics and place of death still remain, or appear to be cancelled out by the home care setting.

\section{STUDIES INVESTIGATING CANCER PATIENTS' LIKELIHOOD OF BEING REFERRED TO PALLIATIVE HOME CARE}

The studies reviewed make one of three types of comparison (Table 3): patients referred to hospices which provide home care only are compared with patients referred to hospices with beds (which may also provide some home care), patients referred to the home care branch of a hospice are compared with those referred to its inpatient branch, or patients referred to home care are compared with some or all of the remaining terminal cancer population. Results from these comparisons will all be reported in the form of patients' likelihood of being referred to home care and it will be assumed that being referred to the home care "arm" in each comparison represents an advantage in achieving home death. 
Studies involving patients both with and without a primary carer found that having a primary carer, being married or living with someone increased the likelihood of being referred to palliative home care (McCusker, 1985; Komesaroff et al., 1989; Dunphy and Amesbury, 1990; Costantini et al., 1993; Bradshaw, 1993). Having a spouse as the primary caregiver also increased likelihood of referral to home care compared to other caregivers (McCusker, 1985). Studies which considered only patients with a primary carer, found that referral to home care was less likely if the carer did not live with the patient or was employed (Mor and Hiris, 1983; Mor et al., 1985; Greer et al., 1986; Powers and Burger, 1987) or old (Mor and Hiris, 1983). Referral to home care was less likely if the carer was male, even when carer employment was considered in the analysis (Mor et al., 1985).

Most studies involving patients both with and without a primary carer indicate that older patients are less likely to be referred to home care (Evans and McCarthy, 1984; McCusker, 1985; Komesaroff et al., 1989; Dunphy and Amesbury, 1990; Costantini et al., 1993; Eve et al., 1997; Talmi et al., 1997) or to hospice care in general (Hunt and McCaul, 1996). Only Sessa et al. (1996) found no age effect. Addington-Hall et al. (1998) have found that patients over age 85 are also less likely to be referred to hospice inpatient care than younger patients. Therefore older patients may fail to access services overall, as suggested by Cartwright (1993). For studies involving only patients with a primary carer, age effects were less clear (Mor et al., 1985; Greer et al., 1986; Powers and Burger, 1987). Mor et al. (1985) found no effects of age. Greer et al. (1986) and Powers and Burger's (1987) results suggest that patients under 65 years of age were more likely to be referred to conventional care compared to older patients. However, conventional care patients had the highest study refusal rates and those who refused were significantly more likely to be old. Therefore the conventional care sample may be younger as an artefact of sample recruitment.

Studies which found a gender effect paradoxically found that females were more likely to be referred to home care than men (Mor et al., 1985; McCusker and Stoddard, 1987; Dunphy and Amesbury, 1990) while men, as shown above, are more likely to die at home. However, most studies report no effect of gender (Evans and McCarthy, 1984; McCusker, 1985; Costantini et al., 1993; Sessa et al., 1996; Eve et al., 1997; Talmi et al., 1997).

Patients referred to home care tended to have a better level of function and fewer nursing care requirements than those referred to inpatient care (Mor and Hiris, 1983; Mor et al., 1985; Greer et al., 1986; Powers and Burger, 1987; Bradshaw, 1993). Home care patients also had longer survival from admission or greater length of stay within the service than patient admitted to inpatient care
(Greer et al., 1986; Powers and Burger, 1987; Komesaroff et al., 1989; Dunphy and Amesbury, 1990; Talmi et al., 1997). This may relate to level of function upon admission. Patients referred to home care (Gray et al., 1987; McCusker and Stoddard, 1987) and to hospice care in general (Hunt and McCaul, 1996) tended to have longer survival from diagnosis compared with other patients. Similarly, longer contact with the local oncology centre was positively related to referral to home care (Sessa et al., 1996). McCusker (1985) found that patients who used home care compared with those who did not, had a longer terminal care period (over 45 days), as reflected by evidence of progressive malignancy and a switch towards palliative rather than curative treatment.

Patients with haematological malignancy were less likely to receive home care (Evans and McCarthy, 1984; McCusker and Stoddard, 1987; Sessa et al., 1996) or hospice care in general (Hunt and McCaul, 1996). Likewise home care was less likely for patients with cancers of the central nervous system (CNS) (Dunphy and Amesbury, 1990). Lung cancer patients tended to be referred to home care (Evans and McCarthy, 1984; Dunphy and Amesbury, 1990; Costantini et al., 1993) and this care location was also associated with a greater incidence of dyspnoea and anxiety or depression upon presentation (Dunphy and Amesbury, 1990). An American study (McCusker and Stoddard, 1987) and an Italian study (Costantini et al., 1993) found that breast cancer tended to be associated with home care, while a U.K. study (Evans and McCarthy, 1984) and a Swiss study (Sessa et al., 1996) suggest the opposite trend. Patients with genitourinary (McCusker and Stoddard, 1987; Costantini et al., 1993) and oropharyngal cancers (Evans and McCarthy, 1984) were more likely to be referred to home care, while patients with gastrointestinal cancers were less likely (Evans and McCarthy, 1984). Among patients with head and neck cancer oral cavity tumours were negatively associated with home care (Talmi et al., 1997).

Referral to home care was positively related to professional and non-manual occupations (Komesaroff et al., 1989), higher income (Greer et al., 1986) and higher education (Costantini et al., 1993). Tables presented by Mor and Hiris (1983) also suggest that home care patients were more likely to be college educated and have higher family incomes compared to inpatients. Greer et al. (1986) found that patients with income below $\$ 10,000$ were less likely both to be referred to home care and conventional care compared to care in hospice with beds.

Talmi et al. (1997) found that pain severity at the onset of care was negatively associated with home care. Powers and Burger (1987) report that patient appetite change, cold sweats, calmness and happiness and carer stress, time commitment, loss of 
income, perceived patient burden and happiness were associated with home care, while the patient being lonely and frightened was associated with other care. However, these factors may have been measured subsequent to admission to care. Powers and Burger (1987) report that patient weight loss was associated with home care while Talmi et al. (1997) report the opposite.

Perhaps not surprisingly the patient's care location towards the end was related to home care use. McCusker (1985) found that patients who spent most of their terminal care period at home were more likely to be home care users. Those not referred to home care tended to have spent more time in hospital towards the end of life (Gray et al., 1987; Sessa et al., 1996). However, one cannot assess whether patients' location was affected by home care or whether use of home care was affected by patients' location.

In summary, many of the factors increasing likelihood of referral to palliative home care are similar to the factors increasing likelihood of place of death. Presence of a primary carer, preferably not male, old or employed, and membership of a high socioeconomic group overall increase likelihood of referral to home care. Being old, having high care requirements or having a haematological malignancy appear to reduce the likelihood.

However, studies in which gender was found to have an effect, report that females tended to be referred to home care, while men tend to die at home. Two of these three studies considered females under the age of 65 (McCusker and Stoddard, 1987) or found a significant effect for young women (Dunphy and Amesbury, 1990). This may reflect a support service bias towards wanting to keep women in a family care setting for as long as possible, perhaps particularly if there are young children present. However, it is not necessarily seen as equally desirable that the death itself should occur at home.

For survival and place of death it appeared that the important difference was whether the diagnosis had been made more or less than 1 month before death. For referral to palliative home care it appeared to be survival from diagnosis in the longer term which was of importance. Survival from diagnosis is not equivalent to length of care period. Longer survival may mean that patients have longer time to establish a relationship with local health care professionals and adjust to the disease. Thus longer survival may make access to home care more likely, while the eventual place of death may be determined by factors other than survival, such as eventual nursing care requirements and length of terminal phase.

\section{STUDIES INVESTIGATING PLACE OF DEATH FOR} CANCER PATIENTS UNDER PALLIATIVE HOME CARE

As the same variables often predict referral to home care and dying at home, it may be admission to palliative home care rather than these variables per se which affects place of death. This section considers place of death of patients who have been admitted to home care, to investigate whether these variables still have an impact on place of death or appear to be cancelled out by home care. Gender showed no relationship or an opposite relationship with home care admission compared with place of death. Therefore, the behaviour of this variable for home care patients will be of particular interest. Unfortunately survival was not reported in the studies below and socioeconomic variables rarely considered.

Studies reviewed (Table 4) again show that patients with a primary carer (McCusker and Stoddard, 1987; Dunphy and Amesbury, 1990; Loven et al., 1990; Hinton, 1994; De Conno et al., 1996) and additional home support (McWhinney et al., 1995) were more likely to die at home. Hays (1986), however, found no differences between patients requiring both home and inpatient care and those requiring home care only and GrothJuncker and McCusker (1983) found no effect of being married or living alone. Likelihood of home death depended on the primary carer's fitness (Hinton, 1994). Carer fatigue, strain, anxiety and depression were negatively associated with home death (Hays, 1986; Hinton, 1994, 1996). Patients who eventually died as inpatients were likely to spend longer time in care (Groth-Juncker and McCusker, 1983; Hinton, 1994) and experience lower quality of life (Hinton, 1994) while reported satisfaction with support was associated with home death (Hinton, 1994). These variables are likely to be associated with the strain placed on the carer during the care period.

Once in home care, age was generally not found to be related to place of death. Only McCusker and Stoddard (1987) report that home care patients who stopped using home care in the last month of life were older than those who remained in home care. Their sample was limited to patients under 65 and not all of their home care patients would eventually die at home.

Likewise once in home care, most studies found no evidence that gender had an effect on place of death. Only McCusker and Stoddard (1987) found that females were more likely to stop receiving home care in the last month of life compared with males and De Conno et al. (1996) found that females were less likely to die at home. The only study which considered socioeconomic factors for home care patients, report that poor financial conditions were negatively associated with death at 
home, while housing conditions and education had no effect (De Conno et al., 1996).

Unrelieved pain was often associated with inpatient admission (Hays, 1986; Dunphy and Amesbury, 1990; Hinton, 1996), likewise psychological symptoms, weakness, peak of any symptom (Hinton, 1994, 1996), nausea/vomiting and respiratory distress (Hays, 1986). The only effects of diagnosis were reported by Hinton (1994) who found that breast cancer patients were more likely to die in inpatient care, while patients with stomach or bowel cancer were more likely to die at home. While the patient's denial of illness was negatively associated with home death (Hinton, 1994), the patient's preference for dying at home was positively associated with death at home (GrothJuncker and McCusker, 1983; McWhinney et al., 1995).

Age and sex may have less impact on home deaths once under home care. However, the presence of a primary carer, the carer's fitness and the strain placed on him or her remained important. The need for symptom control clearly emerged as a key variable in inpatient admissions. Other research investigating reasons for final inpatient admissions, without comparison with non-admitted patients, have also identified symptom control as a major reason for admission (Herd, 1990) alongside insufficient care resources in the home (Doyle, 1980; Wilkes, 1984; Herd, 1990).

\section{SUMMARY AND DISCUSSION}

Research reviewed in this paper shows that both the likelihood of dying at home and of being admitted to palliative home care depend on patient characteristics. Some of these characteristics relate to clinical variables and support requirements and may justify differential treatment. However, other variables suggest that the opportunity to access home care and achieving home death are unevenly distributed.

During this century there has been a decrease in the proportion of deaths occurring at home, at least in the developed world. This occurred in parallel with an increased belief in and availability of hospital interventions and a decrease in family unit size and increase in life expectancy. This meant that there was a decrease in family support available and an increase in the proportion of older people in need of care and that patients more often tended to die in a technical, hospital environment. Increasing recognition of the inadequacy of acute hospital care in meeting the needs of the dying, led to the rise of the modern hospice movement, beginning with $\mathrm{St}$ Christopher's Hospice in the U.K. in 1967. Similar developments of hospice care occurred in U.S.A. in the 1970s (Siebold, 1992) and Australia in the 1980s (Hunt et al., 1991). A natural extension of this approach was to support patients in their own home environment, and there was a rapid expansion of home care teams in the U.K. in the early 1980s (Boyd, 1994), while home care developed more in parallel with inpatient care in U.S.A. and Australia. Furthermore, there was increased focus on improved palliation of symptoms. The proportion of deaths in inpatient hospices have risen in accord with service developments (Seale and Cartwright, 1994). Similarly, as we have seen, home care services appear to increase the number of deaths at home, although it is more difficult to establish a direct link. However, although these services may improve the care of cancer patients, this group still display similar patterns of inequality in terms of age and sex as other groups and in addition appear to display differences in access and place of death based on socioeconomic factors. Therefore where improvements in palliative care occur, these may not reach everybody in the same diagnostic group equally and may in fact exacerbate differences between patients.

The literature on place of death has mainly emerged subsequent to the rise of the hospice movement. All but two studies reviewed in this paper were published from 1980 onwards. The studies reviewed here consider different settings and focus on different variables and as such the conclusions drawn must be viewed with some caution. Furthermore the review has focused on studies from North America, Europe and Australia and especially Anglo-Saxon countries. Thus its findings are restricted to these cultures over the last, few decades. The situation during earlier periods and in developing countries is bound to be very different. The patterns emerging within the scope of the review, however, seem quite consistent, particularly in relation to informal support, age, sex and socioeconomic variables.

Significant relationships were found for a number of clinical variables. Symptom control needs were an important factor in inpatient admission. This highlights the need for improved symptom control in the community. Survival from diagnosis of less than 1 month was negatively associated with home death. Longer term survival was positively associated with referral to home care. Longer survival may give more time to establish links with community support and come to terms with illness. This may indicate the value of early, rather than late, referral to home care services. Haematological and CNS cancers were both negatively associated with home death and home care. Haematological cancers presumably require more hospital intervention and both patients and staff may want to continue the relationship they have developed over many months. The changes associated with CNS cancers may place more strain on carers. Gastrointestinal and genitourinary cancers were positively related to home death. The latter was also positively related to home care while the former was negatively re- 
lated. Lung and head/neck cancer patients were less likely to die at home but more likely to be referred to home care. Although lung cancer is to date typically a male cancer (because of smoking behaviour) it shows an opposite relationship to home death and home care to that otherwise observed for men. The relationship between breast cancer and referral to home care is unclear, but it appears negatively associated with place of death for home care patients. Being predominantly a female cancer, gender may contribute to the patterns observed. Different cancers are associated with different courses of illness, survival and gender distributions. An understanding of the interaction between these variables and place of death would require research in greater detail on a larger patient sample than has hitherto been attempted.

Four non-clinical variables showed a relationship with home deaths and access to home care: presence of primary carer, age, gender and socioeconomic status. The presence of a primary carer and the strain placed on him/her were key variables in relation to home deaths. Home care support did not remove this relationship. Health professionals may be more likely to refer patients to home care when there are more care resources in the home. However, even from this position of strength, in which professional home care is added to informal care resources, death at home is still highly dependent on the carers' ability to stay the course. Although the patient is defined as the client, there may need to be a substantial shift in focus towards the needs of the patient's carer, if there is to be an improvement in proportion of home deaths.

Older people are less likely to die at home. This may be because they are less likely to have a suitable primary carer and more likely to have additional care needs and to reside in nursing homes. However, it may also relate to their poorer access to home care, as well as to services in general (Cartwright, 1993; Addington-Hall et al., 1998). The review suggests that once in home care older people do not tend to be less likely to die at home. Although more information is required on the characteristics of older patients referred to home care, it is possible that better access to home care services would help towards removing the bias against home death in old age.

Women were less likely to die at home than men. However, they were not less likely to access home care and younger women may even be more likely to receive home care support than men. Thus women are not disadvantaged in their access to home care and causes for their disadvantage in achieving home death need to be sought elsewhere. Gender roles may explain the patterns observed, although effects of age need to be considered. In relation to home care there may be a greater tendency to call in help when males are the carers, while female carers may more often be left to cope. There may also a greater urgency to maintain the female patient in the family context, particularly when the family has young children. In relation to home deaths, the studies reviewed suggest that men are less effective as carers and past research shows that the greater burden of care typically falls on women (Anderson, 1987). As home deaths are greatly dependent on carers' ability to cope, any differences in ability to fulfil the carer role are likely to have considerable impact. While home care may redress the gender imbalance for those under such care, improvements in home death for women overall may require helping men to take on and cope with the carer role to a larger extent.

While primary carer support, age and sex showed a similar relationship to home death both for cancer and non-cancer patients, socioeconomic factors were associated with home death for cancer patients only. Higher socioeconomic groups were more likely to die at home. This is likely to be related to better access to support as these groups also appeared more likely to be referred to home care. However, present data do not allow us to assess directly whether being in home care helped redress the imbalance in home deaths for lower socioeconomic groups. Higher socioeconomic groups are better able to pay for additional care and may be more effective at gaining access to the services available to cancer patients, e.g. through skills associated with higher education. As the range of support available for cancer is greater, the difference between those who are effective at gaining access and those who are not may also be greater. This suggests the need to improve cancer service access for lower socioeconomic groups by a targeted approach. It similarly suggests that any expansion of cancer services to other patient groups needs to take care not to extend socioeconomic inequalities in place of death among cancer patients to other patient groups.

The studies reviewed were conducted alongside rising interest in care for the dying. Place of death is commonly included in palliative care research as it is one of its few measurable outcomes, with home death typically considered to be a positive outcome. Patient morbidity is arguably less applicable in this field and patients' own assessments of symptoms and quality of life are often difficult to obtain in the terminal phase. However, whilst factors relating to place of death therefore have been routinely analysed in numerous studies, researchers have not been particularly concerned with the inequalities to which these studies point, nor with designing further research to understand why these patterns emerge.

More work is required to understand the access to home care and its role in place of death. Most studies of access to home care consider patients already referred to hospice or specialist care, who therefore may already be subject to the biases of 
interest in this review (Mor and Hiris, 1983; Mor et al., 1985; Greer et al., 1986; Powers and Burger, 1987; Komesaroff et al., 1989; Dunphy and Amesbury, 1990; Bradshaw, 1993; Eve et al., 1997). Geographical distance to services also needs to be taken into account. For instance, in some studies utilising National Hospice Study data patients did not have equal geographical access to home or inpatient hospice care (Mor and Hiris, 1983; Greer et al., 1986; Powers and Burger, 1987). Furthermore a proper assessment of the impact of home care on home deaths requires carefully controlled studies which take into account the type of home care and the proportion of patients in the population accessing home care.

The retrospective, quantitative data collected from patient records and official statistics often used in this area of research are necessary to establish what the overall patterns are. Such routinely collected data can be further utilised to investigate how variables such as age and sex, marital and socioeconomic status may interrelate. However, to understand why a variable affects home care access and place of death, a more detailed and labour intensive approach is now required. For instance, we need to establish whether it is socioeconomic area of residence or an individual's education, income or occupation which affects outcomes. The former would suggest that more services simply should be provided in deprived areas, the latter that we need to understand the mechanisms whereby individuals acquire help. We need to establish how behaviour and perceptions of roles may differ depending on sex and how patients' age may affect attitudes and decision making. We need to investigate the circumstances under which informal carer support breaks down and when it does not. This requires more labour intensive research methods, including prospective observation and semi-structured interviews. The longitudinal research conducted by Hinton $(1994,1996)$ is a step in the right direction.

Until we understand the mechanisms behind differences in access to home care and deaths at home, further general improvements in home care support may only help those who are already at an advantage, in accord with the inverse care law (Tudor-Hart, 1971). Increased support may therefore not have an impact on home deaths which corresponds to resources invested. If more deaths at home is the aim, real progress may only be achieved by identifying why certain groups currently are at a disadvantage and targeting interventions towards removing these disadvantages. Until such time we will be no more equal in death than we have been in life.

Acknowledgements - The work undertaken for this review was made possible through funding from the Elizabeth Clark Charitable Trust and the NHS R\&D Programme
Primary/Secondary Care Interface, although the views expressed are the authors' and may not be those of either organisation.

\section{REFERENCES}

Addington-Hall, J. M., Altmann, D. and McCarthy, M (1998) Which terminally ill patients receive hospice inpatient care? Social Science and Medicine 46(8), 10111016.

Addington-Hall, J. M., MacDonald, L. D., Anderson, H. R. and Freeling, P. (1991) Dying from cancer: The views of bereaved family and friends about the experiences of terminally ill patients. Palliative Medicine 5, 207-214

Anderson, J. T. (1987) The unremitting burden on carers. British Medical Journal 294, 73-74.

Aries, P. (1981) The Hour of our Death. Penguin, Harmondsworth.

Ashby, M. and Wakefield, M. (1993) Attitudes to some aspects of death and dying, living wills and substituted health care decision-making in South Australia: public opinion survey of a parliamentary select committee. Palliative Medicine 7, 273-282.

Axelsson, B. and Christensen, S. B. (1996) Place of death correlated to sociodemographic factors: a study of 203 patients dying of cancer in a rural Swedish county in 1990. Palliative Medicine 10, 329-335.

Bowling, A. and Cartwright, A. (1982) Life after Death: A Study of the Elderly Widowed. Tavistock, London.

Boyd, K. J. (1994) Hospice home care in the United Kingdom. Annals Academy of Medicine Singapore 23(2), 271-274.

Bradshaw, P. J. (1993) Characteristics of clients referred to home, hospice and hospital palliative care services in Western Australia. Palliative Medicine 7, 101-107.

Cartwright, A. (1991) Balance of care for the dying between hospitals and the community: perceptions of general practitioners, hospital consultants, community nurses and relatives. British Journal of General Practice 41(348), 271-274.

Cartwright, A. (1993) Dying when you're old. Age and Ageing 22, 425-430.

Cartwright, A., Hockey, L. and Anderson, J. L. (1973) Life Before Death. Routledge and Kegan Paul, London.

Catalan-Fernandez, J. G., Pons-Sureda, O., RecoberMartinez, A., Avella-Mestre, A., Carbonero-Malberti, J. M., Benito-Oliver, E. and Garau-Llinas, I. (1991) Dying of cancer. The place of death and family circumstances. Medical Care 29(9), 841-852.

Charlton, R. C. (1991) Attitudes towards care of the dying: a questionnaire survey of general practice attenders. Family Practice 8(4), 356-359.

Clifford, C. A., Jolley, D. J. and Giles, C. G. (1991) Where people die in Victoria. Medical Journal of Australia 155, 446-456.

Costantini, M., Camoirano, E., Madeddu, L., Bruzzi, P., Verganelli, E. and Henriquet, F. (1993) Palliative home care and place of death among cancer patients: a population-based study. Palliative Medicine 7, 323-331.

De Conno, F., Caraceni, A., Groff, L., Brunelli, C., Donati, I., Tamburini, M. and Ventafridda, V. (1996) Effect of home care on the place of death of advanced cancer patients. European Journal of Cancer 32A(7), $1142-1147$.

Doyle, D. (1980) Domiciliary terminal care. Practitioner 224, 575-582.

Dunlop, R. J., Davies, R. J. and Hockley, J. M. (1989) Preferred vs actual place of death: a hospital palliative care support team experience. Palliative Medicine $\mathbf{3}$, 197-201. 
Dunphy, K. P. and Amesbury, B. D. V. (1990) A comparison of hospice and home care patients: patterns of referral, patient characteristics and predictors of place of death. Palliative Medicine 4, 105-111.

Evans, C. and McCarthy, M. (1984) Referral and survival of patients accepted by a terminal care support team. Journal of Epidemiology and Community Health 38, 310314.

Eve, A., Smith, A. M. and Tebbit, P. (1997) Hospice and palliative care in the U.K. 1994-1995, including a summary of trends 1990-1995. Palliative Medicine 11, 3143.

Gray, D., MacAdam, D. and Boldy, D. (1987) A comparative cost analysis of terminal cancer care in home hospice patients and controls. Journal of Chronic Disease 40(8), 801-810.

Greer, D. S., Mor, V., Morris, J. N., Sheerwood, S., Kidder, D. and Birnbaum, H. (1986) An alternative in terminal care: results of the National Hospice Study. Journal of Chronic Disease 39(1), 9-26.

Groth-Juncker, A. and McCusker, J. (1983) Where do elderly patients prefer to die? Place of death and patient characteristics of 100 elderly patients under the care of a home health care team Journal of the American Geriatrics Society 31(8), 457-461.

Hays, J. C. (1986) Patient symptoms and family coping: predictors of hospice utilization patterns. Cancer Nursing 9(6), 317-325.

Herd, E. B. (1990) Terminal care in a semi-rural area. British Journal of General Practice 40, 251-258.

Higginson, I., Webb, D. and Lessof, L. (1994) Reducing hospital beds for patients with advanced cancer. Lancet 344(8919), 409.

Hinton, J. (1994) Which patients with terminal cancer are admitted from home care? Palliative Medicine 8, 197210.

Hinton, J. (1996) Services given and help perceived during home care for terminal cancer. Palliative Medicine 10, $125-134$.

Hunt, R. W., Bond, M. J., Groth, R. K. and King, P. M. (1991) Place of death in South Australia. Patterns from 1910 to 1987. Medical Journal of Australia 155, $549-553$.

Hunt, R., Bonett, A. and Roder, D. (1993) Trends in the terminal care of cancer patients: South Australia, 18981990. Australia and New Zealand Journal of Medicine 23(3), 245-251

Hunt, R. and McCaul, K. (1996) A population-based study of the coverage of cancer patients by hospice services. Palliative Medicine 10, 5-12.

Hunt, R., Roder, D. and MacHarper, T. (1989) The impact of hospice services on places of death of South Australians. Cancer Forum 13, 110-113.

Johnson, H. and Oliver, D. (1991) The development of palliative care services and the place of death of cancer patients. Palliative Medicine 5, 40-45.

Komesaroff, P. A., Moss, C. K. and Fox, R. M. (1989) Patients' socioeconomic background: influence on selection of inpatient of domiciliary hospice terminal-care programmes. Medical Journal of Australia 151, 196-201.

Loven, D., Golberg, E., Hart, Y. and Klein, B. (1990) Place of death of cancer patients in Israel: the experience of a "home-care programme". Palliative Medicine 4, 299-304.

McCusker, J. (1983) Where cancer patients die: an epidemiologic study. Public Health Reports 98(2), 170-178.

McCusker, J. (1985) The use of home care in terminal care. American Journal of Preventive Medicine 1(2), 4252.

McCusker, J. and Stoddard, A. M. (1987) Effects of an expanding home care program for the terminally ill. Medical Care 25(5), 373-385.
McWhinney, I. R., Bass, M. J. and Orr, V. (1995) Factors associated with location of death (home or hospital) of patients referred to a palliative care team. Canadian Medical Association Journal 152(3), 361-367.

Moinpour, C. M. and Polissar, L. (1989) Factors affecting place of death of hospice and non-hospice patients. American Journal of Public Health 79(11), 1549-1551.

Mor, V. and Hiris, J. (1983) Determinants of site of death among hospice cancer patients. Journal of Health and Social Behaviour 24, 375-385.

Mor, V., Wachtel, T. J. and Kidder, D. (1985) Patient predictors of hospice choice: hospice vs home care programs. Medical Care 23, 1115-1119.

Parkes, C. M. (1978) Home or hospital? Terminal care as seen by surviving spouses Journal of the Royal College of General Practitioners 28, 19-30.

Polissar, L., Severson, R. K. and Brown, N. K. (1987) Factors affecting place of death in Washington State, 1968-1981. Journal of Community Health 12(1), 40-55.

Powers, J. S. and Burger, M. C. (1987) Terminal care preferences: hospice placement and severity of disease. Public Health Reports 102(4), 444-449.

Roder, D., Bonett, A., Hunt, R. and Beare, M. (1987) Where patients with cancer die in South Australia. Medical Journal of Australia 147, 11-13.

Rosenberg, E. and Short, C. (1983) Issues of institutionalization: $5 \%$ fallacies and terminal care. International Journal of Aging and Human Development 17(1), 43-55.

Sanderson, H., Mountney, L. and Harris, J. (1992) Epidemiologically Based Needs Assessment Report 11: Cancer of the Lung. NHS Management Executive, London.

Seale, C. (1991) Death from cancer and death from other causes: the relevance of the hospice approach. Palliative Medicine 5, 12-19.

Seale, C. and Cartwright, A. (1994) The Year Before Death. Avebury, Aldershot.

Sessa, C., Roggero, E., Pampallona, S., Regazzoni, S., Ghielmini, M., Lang, M., Marx, B., Neuenschwander, H., Pagani, O., Vasilievic, V. and Cavalli, F. (1996) The last 3 months of life of cancer patients: medical aspects and role of home-care services in southern Switzerland. Support Care Cancer 4(3), 180-185.

Siebold, C. (1992) The Hospice Movement: Easing Death's Pains. Twayne Publishers, New York.

Sims, A., Radford, J., Doran, K. and Page, H. (1997) Social class variation in place of cancer death. Palliative Medicine 11, 369-373.

Smith, A. M., Eve, A. and Sykes, N. P. (1992) Palliative care services in Britain and Ireland 1990: an overview. Palliative Medicine 6, 277-291.

Talmi, Y. P., Bercovici, M., Waller, A., Horowitz, Z., Adunski, A. and Kronenberg, J. (1997) Home and inpatient hospice care of terminal head and neck cancer patients. Journal of Palliative Care 13(1), 9-14.

Toscani, F., Cantoni, L., Di Mola, G., Mori, M. Santosuosso, A. and Tamburini, M. (1991) Death and dying: perceptions and attitudes in Italy. Palliative Medicine 5, 334-343.

Townsend, J., Frank, A. O., Fermont, D., Dyer, S., Karran, O., Walgrove, A. and Piper, M. (1990) Terminal cancer care and patients' preference for place of death: a prospective study. British Medical Journal 301, 415-417.

Tudor-Hart, J. (1971) The inverse care law. Lancet 1(696), $405-412$

Ward, A. W. M. (1987) Home care services: an alternative to hospices? Community Medicine 9(1), 47-54.

Wilkes, E. (1984) Dying now. Lancet 1(8383), 950-952.

Zimmer, J. G., Groth-Juncker, A. and McCusker, J. (1984) Effects of a physician-led home care team on terminal care. Journal of the American Geriatrics Society 32, 288293. 